\title{
Crystal structure, DNA binding studies, nucleolytic property and topoisomerase I inhibition of zinc complex with 1,10-phenanthroline and 3 methyl-picolinic acid
}

\begin{abstract}
Crystal structure analysis of the zinc complex establishes it as a distorted octahedral complex, bis(3-methylpicolinato-ə2 N,O)2(1,10-phenanthroline-ə2 N,N)-zinc(II) pentahydrate, [Zn(3Me-pic)2(phen)] $5 \mathrm{H} 2 \mathrm{O}$. The trans-configuration of carbonyl oxygen atoms of the carboxylate moieties and orientation of the two planar picolinate ligands above and before the phen ligand plane seems to confer DNA sequence recognition to the complex. It cannot cleave DNA under hydrolytic condition but can slightly be activated by hydrogen peroxide or sodium ascorbate. Circular Dichroism and Fluorescence spectroscopic analysis of its interaction with various duplex polynucleotides reveals its binding mode as mainly intercalation. It shows distinct DNA sequence binding selectivity and the order of decreasing selectivity is ATAT > AATT > CGCG. Docking studies lead to the same conclusion on this sequence selectivity. It binds strongly with G-quadruplex with human tolemeric sequence 5NAG3(T2AG3)3-3 Njean inhibit topoisomerase I efficiently and is cytotoxic against MCF-7 cell line.
\end{abstract}

Keyword: Zinc(II) ternary complex; Duplex and quadruplex DNA binding; Nucleoytic; Topo I inhibition; Molecular modeling; Docking 